\title{
Subdural spread of medulloblastoma: case report
}

\author{
G. H. KOENIG \\ From the Department of Surgery, Division of Neurosurgery, and Department of Pathology (Neuropathology), \\ Stanford University School of Medicine, Palo Alto, California, U.S.A.
}

SUMMARY A case of widespread intracranial subdural invasion by medulloblastoma is presented. The origin of the metastases would seem to be a large subfrontal tumour mass, with dissemination through the subdural space. This appears to be the first report of intracranial spread of tumour by this route.

Spread of medulloblastoma throughout the ventricular system and subarachnoid space has been amply recorded and well summarized (Zulch, 1956). The finding that intracranial tumours, including medulloblastomas, will also, on occasion, metastasize widely outside the nervous system has been the subject of numerous recent case reports and analyses (Glasauer and Yuan, 1963). A review of the literature prompted by a recently observed case of widespread dural invasion by a medulloblastoma revealed a surprising silence on the subject; this case is therefore reported.

\section{CASE REPORT}

A 2 year old girl was admitted to the Stanford University Hospital after two weeks of unsteady gait and intermittent vomiting. Examination revealed a slightly obtunded, irritable white girl. Pertinent findings included bilateral papilloedema, bilateral dysmetria, an ataxic gait with a tendency to fall in either direction, and a MacEwen's sign. She was scheduled for elective ventriculography and craniotomy, but during the night of admission her pulse rate dropped to $60 / \mathrm{min}$ and she became unrousable. Ventriculography revealed aqueductal compression and the fourth ventricle was not seen. After the placement of a Scott cannula in the right ventricle, a posterior fossa craniectomy was performed which revealed a large jellylike, fragile tumour in the vermis extending into and blocking the ventricle. The infiltrative tumour could not be removed completely and a small portion was left in the vermis. Microscopic examination revealed a classical medulloblastoma.

Postoperatively the patient continued to be irritable and vomited frequently. She had a consistently elevated pressure on ventricular taps. During this time she was noted to be blind. Two weeks after operation a right ventriculoatrial shunt was performed, and six days later a course of irradiation to the whole brain and spinal axis was begun. One week thereafter, a flaccid paraplegia developed with a sensory level at T7-T9 dermatome. The irradiation, which had included the low spinal subarachnoid space, was discontinued, and the patient was discharged. She improved slightly, then proceeded on a downhill course. She died two months after her discharge from the hospital and four months after herr operation.

PATHOLOGY The necropsy performed in another institu踝 tion was limited to the central nervous system. The brain spinal cord, and dura mater were removed en bloc and together weighed $1,290 \mathrm{~g}$. The dura mater of the cerebrab convexity was covered on its inner surface with multip通 $\stackrel{\rho}{9}$ nodules of whitish firm tumour, which extended along. the falx deeply between the hemispheres (Figs. 1 and 2$)$ The largest nodule measured 2.0 by $2.5 \mathrm{~cm}$. The lepto- .meningeal coverings of the cerebral hemispheres were unremarkable, but on separating them multiple large nodules were seen between the hemispheres and in the corpus callosum. The inferior surface of the cerebrum revealed an almost continuous sheet of subarachnoid tumour which extended from the frontal tip to the optic chiasm and measured 5.1 by $5.9 \mathrm{~cm}$ (Fig. 3). On coronal sections, nodules of tumour measuring up to 3.0 by $1.7 \mathrm{~cm}$ were noted in the parenchyma of the right frontal lobe and throughout the corpus callosum, as well as a small mass measuring 1.0 by $0.6 \mathrm{~cm}$ in the left globus pallidus. In horizontal sections of the cerebellum and brain-stem there was a 1.5 by $0.7 \mathrm{~cm}$ area of softening which presented a puckered appearance in the operative $\dot{T}$ site in the cerebellar vermis, but no obvious gross residual tumour was found.

Over the dorsal surface of the spinal cord, several small nodules of subarachnoid growth were encountered which compressed but did not infiltrate the cord. Microscopically, the tumour was characterized by extreme $D$ cellularity, with scanty cytoplasm and densely haematoxophilic round or oval nuclei. Mitotic figures were numerous. N No rosettes were seen. When stained with silver for 0 reticulin, the tumour demonstrated a lobular pattern. $N$ The appearances were typical of medulloblastoma $\underset{\mathrm{N}}{\mathrm{N}}$ (Fig. 4). 


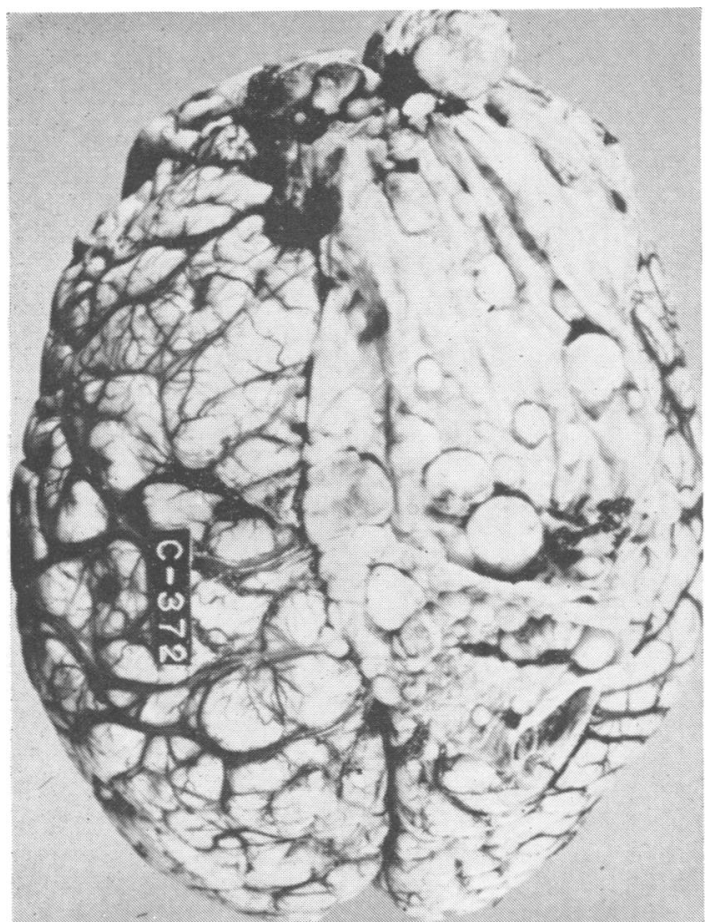

FIG. 1. Dura mater of left hemisphere reflected, demonstrating multiple nodules on inner surface without involvement of underlying leptomeninges or brain parenchyma.

Examination of the cerebellum revealed dense fibrillary gliosis in the vermis, with several residual microscopic nests of tumour.

\section{DISCUSSION}

Seeding of a medulloblastoma has been known for at least 30 years (Bailey and Cushing, 1925). Likewise, direct extension of tumour into the operative wound and musculature of the neck has been reported (Mittelbach, 1935; Gerlach, 1959; Myake, Toyama, Etani, and Fukuma, 1964). Medulloblastoma seeding into the intracranial dura mater has been noted only as small, scattered nodules limited to the vicinity of the craniectomy site (Friborsky, 1963; Myake et al., 1964).

The mechanism of massive spread of the subdural metastatic lesions over the convexities and the falx in this case is not immediately apparent. While intimately bound to the inner surface of the dura mater, these masses did not appear to have transgressed the underlying leptomeninges, to which they were not adherent. It must be assumed, there-

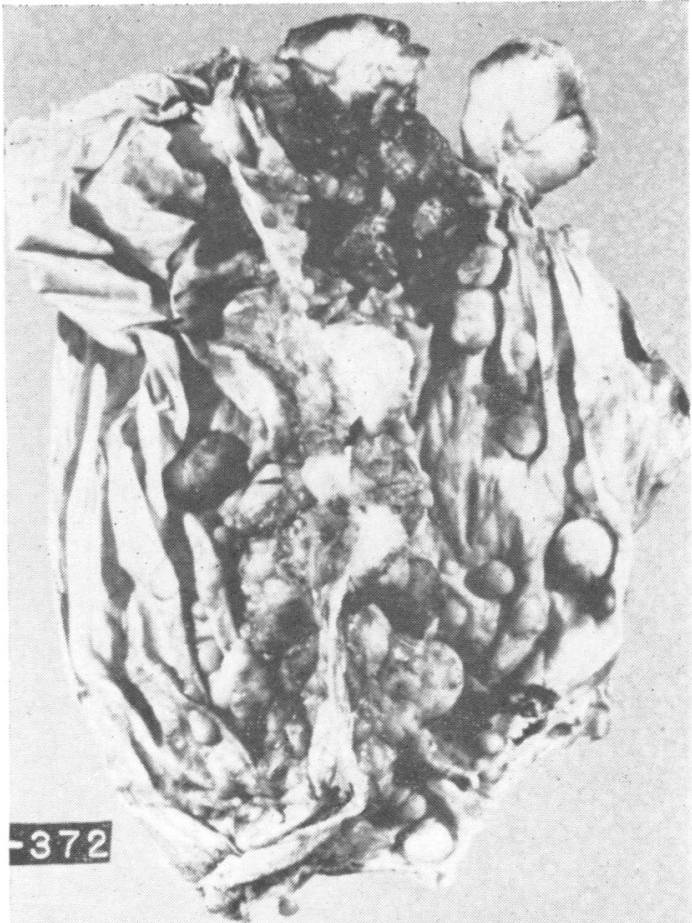

FIG. 2. Inner surface of intracranial dura mater showing nodules of tumour on inner surface.

fore, that they developed after previous dissemination of the tumour cells in the subdural space, presumably from a focus of subarachnoid growth which had transgressed the arachnoid mater at a more remote site. The pathological findings in this case point to the large subfrontal mass as the most likely site of origin of tumour spread. A large subfrontal metastasis, located at the edge of the irradiation field and serving as the probable focus of extracranial metastatic spread has been described by Rubinstein (1959). The subfrontal mass of the present case shares many features with the case of Rubinstein. It is most likely that this subfrontal tumour, penetrating the subdural space, represents the source of medulloblastoma cells which circulated through the subdural space and became adherent to the inner surface of the dura mater. Considering the minimal residual tumour in the cerebellum, the primary tumour site appears an unlikely source of these large masses. The operative interruption of the meninges by the ventriculoatrial shunt is unlikely to be a factor, inasmuch as no tumour was noted along the track of the ventricular catheter. 


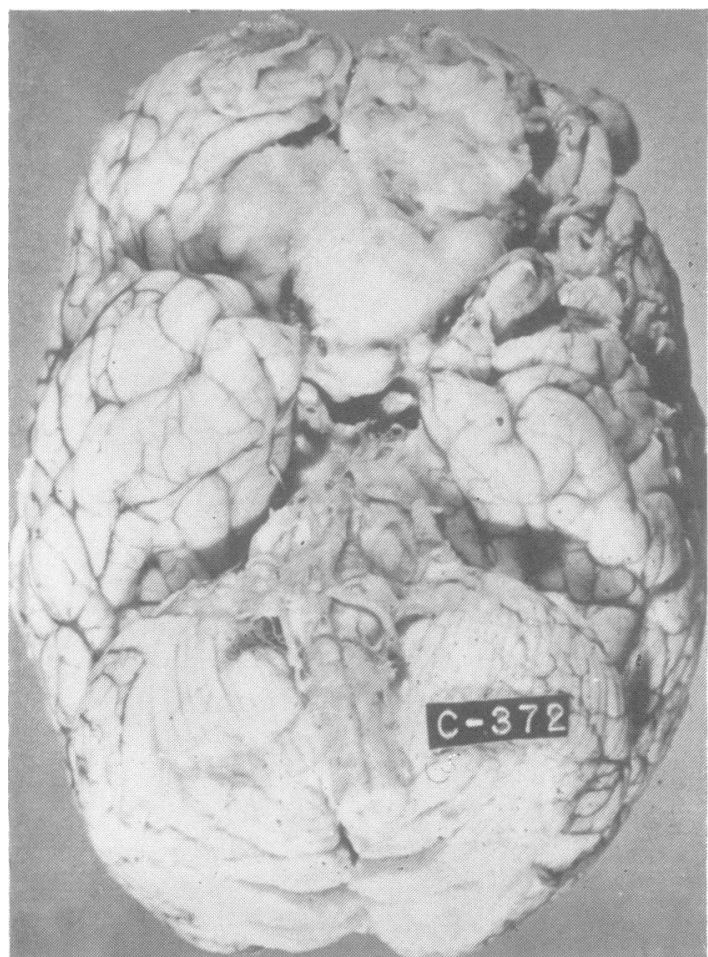

FIG. 3. Subfrontal metastasis extending to but not adherent to optic chiasm.

The author wishes to express his gratitude to Professor L. J. Rubinstein for his encouragement and generous advice. This study was supported by Graduate Training Grant in Neuropathology No. 2-T01-NB-05500-03 from the National Institute of Neurological Diseases and Stroke, U.S. Public Health Service.

\section{REFERENCES}

Bailey, P., and Cushing, H. (1925). Medulloblastoma cerebelli, a common type of midcerebellar glioma of childhood. Arch. Neurol. Psychiat. (Chic.), 14, 192-224.

Friborsky, V. (1963). Medulloblastoma of the cerebellum. Extracranial metastases and diagnostical difficulties. Neoplasm, 10, 427-440.

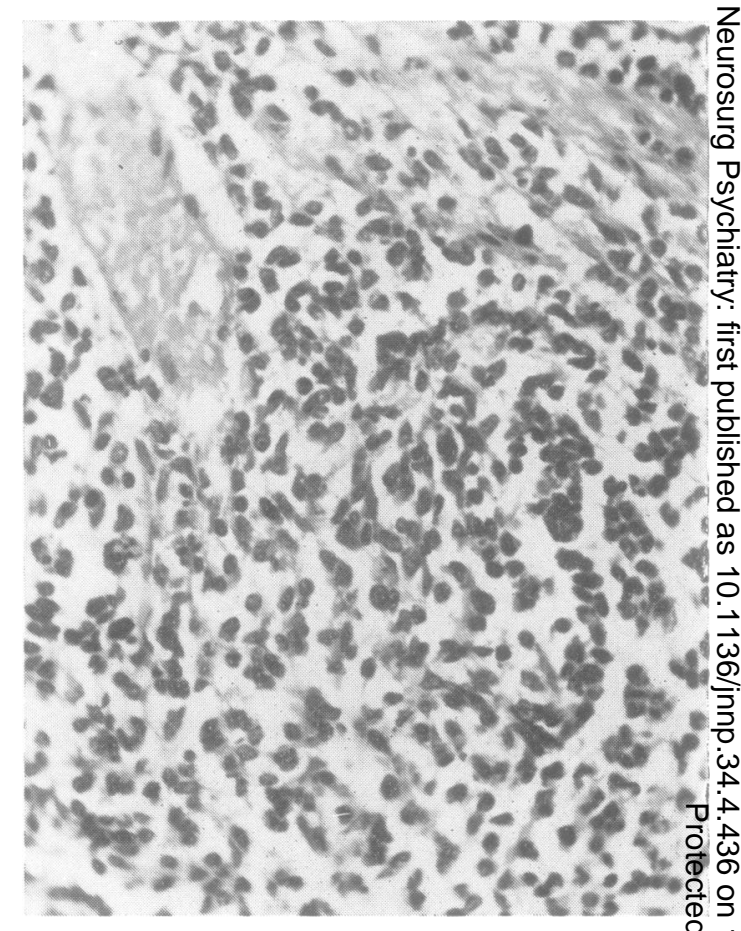

FIG. 4. Representative section of medulloblastoma frome nodule in corpus callosum. $H$ and $E, \times 285$.

Gerlach, J. (1959). Zur Frage der Metastasierung von Hirngeschwülsten in den Körper. Zentrbl. Neurochir., 19, 292-298.

Glasauer, F. E., and Yuan, R. H. P. (1963). Intracranial tumors with extracranial metastases. Case report and $\bar{D}$ review of the literature. J. Neurosurg., 20, 474-493.

Mittelbach, M. (1935). Über Gliome mit Metastasen. Beitr. $\stackrel{\mathbb{Q}}{\mathbb{Q}}$ path. Anat., 95, 538-572.

Miyake, S., Toyama, M., Etani, B., and Fukuma, S. (1964) $\overline{\overline{0}}$ Cerebellar medulloblastoma with postoperative extracran- 3 ial spread. Report of a case. J. Neurosurg., 21, 416-418.

Rubinstein, L. J. (1959). Extracranial metastases in cerebellar medulloblastoma. J. Path. Bact., 78, 187-195.

Zulch, K. J. (1956). Biologie und Pathologie der Hirngeschwülste. In Handbuch der Neurochirurgie, Vol. 3, pp. 131136. Edited by $\mathbf{H}$. Olivecrona and W. Tönnis. Springer:Berlin. 